\title{
Cost-Effective Multiservices Hybrid Access Networks With no Optical Filter at Remote Nodes
}

\author{
Chun-Ting Lin, Po Tsung Shih, Jason (Jyehong) Chen, Peng-Chun Peng, Sheng-Peng Dai, Wen-Jr Jiang,
} Wen-Qiang Xue, and Sien Chi

\begin{abstract}
This letter experimentally demonstrates a multiservices hybrid access network integrated radio-over-fiber and fiber-to-the-home systems which share the same distributed architecture. A $1.25-\mathrm{Gb} / \mathrm{s}$ baseband signal and a 14.375-GHz radio-frequency (RF) signal with $625-\mathrm{Mb} / \mathrm{s}$ binary phase-shift keying data are generated and transmitted employing a commercially available dual-electrode Mach-Zehnder modulator. The proposed scheme offers better performance, has no RF fading issue, can carry vector signals, and requires no optical filter at remote nodes. After transmission over 25-km single-mode fiber, the proposed scheme successfully achieves less than $0.5-\mathrm{dB}$ sensitivity penalties.
\end{abstract}

Index Terms-External modulation, fiber-to-the-home (FTTH), optical access networks, optical communication, radio-over-fiber.

\section{INTRODUCTION}

$\mathbf{T}$ HE FTTx optical access network has increasingly been accepted and deployed as the future-proof broadband services for various applications (e.g., triple-play, telephone, internet,and television). Delivering quad-play services (wireless, telephone, internet, and television) on the installed FTTx network is gaining considerable attention around the world. The convergence of fixed and mobile services on hybrid access networks has great potential to cater multiple services to end users with better choices, superior flexibility, and lower cost [1]-[6]. Recently, simultaneous generation and transmission of wireless radio-frequency (RF) signal and fiber-to-the-home (FTTH) baseband (BB) signal using external modulators has been extensively investigated [1]-[6]. However, [1] and [2] suffer from performance fading issues due to fiber dispersion and interchannel interference between the BB and RF signals. In [3], the BB signal carries identical data to the RF signal and can only employ an ON-OFF-keying (OOK) format. Furthermore, in [4]-[6], more than one narrowband optical filter is needed at the remote nodes and two optical external modulators are needed [6]. To

Manuscript received December 2, 2007; revised January 16, 2008. This work was supported by the National Science Council of the Republic of China, Taiwan, under Contract NSC 96-2221-E-155-038-MY2, Contract NSC 95-2221-E-009-085, Contract NSC 95-2221-E-155-072, Contract NSC 95-2221-E-155-059, Contract NSC 96-2752-E-009-004-PAE, and Contract NSC 96-2628-E-009-016.

C.-T. Lin, P. T. Shih, J. Chen, S. P. Dai, and W.-J. Jiang are with the Department of Photonics, National Chiao Tung University, Hsinchu 300, Taiwan, R.O.C. (e-mail: jinting@ms94.url.com.tw; boris.eo95g@nctu.edu.tw).

P.-C. Peng and W.-Q. Xue are with the Department of Applied Materials and Optoelectronic Engineering, National Chi Nan University, Puli Township, Nantou County 545, Taiwan, R.O.C.

$\mathrm{S}$. Chi is with the Department of Electrical Engineering, Yuan Ze University, Jung-Li, TaoYuan 320, Taiwan, R.O.C.

Color versions of one or more of the figures in this letter are available online at http://ieeexplore.ieee.org.

Digital Object Identifier 10.1109/LPT.2008.921137

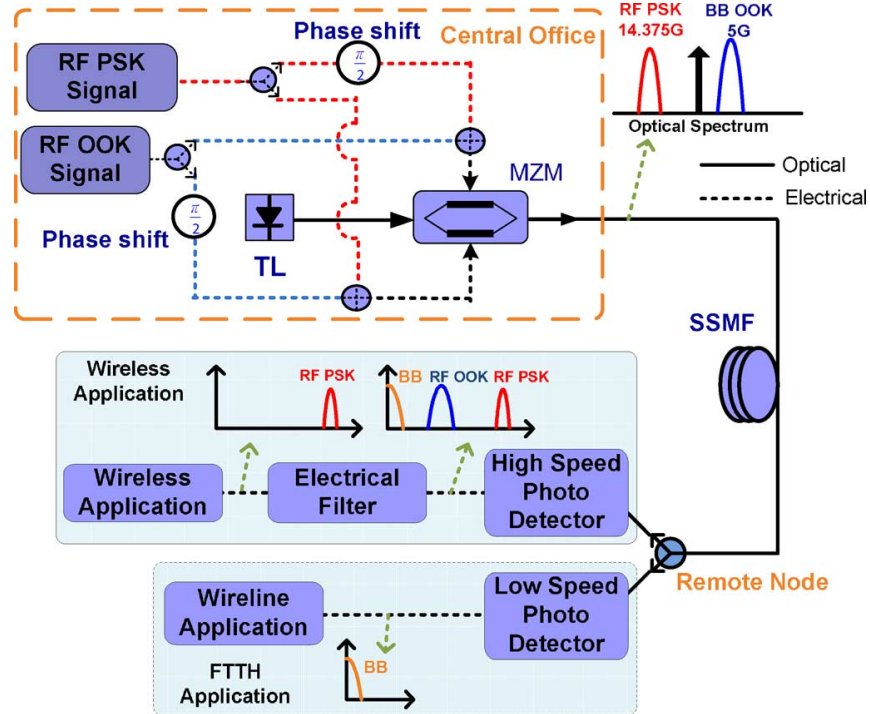

Fig. 1. Conceptual diagram of the proposed system. (TL: tunable laser.)

reduce the cost and complexity, and to overcome the fiber dispersion, it is highly desirable to be able to generate both RF and $\mathrm{BB}$ signals using only a single external modulator, without suffering from RF fading, able to carry vector signals for the wireless applications, and require no optical filtering at remote nodes.

In this letter, we experimentally demonstrate multiservices modulation and fiber-optical transmission of a $1.25-\mathrm{Gb} / \mathrm{s}$ OOK $\mathrm{BB}$ signal and a $625-\mathrm{Mb} / \mathrm{s}$ binary phase-shift keying (BPSK) RF signal on a single wavelength using a commercial external modulator and no optical filters at remote nodes. Furthermore, the proposed scheme does not suffer from the performance fading issue and it can carry vector signals which are of utmost important for wireless applications. Moreover, the proposed system is the first, to our best knowledge, to require no narrowband optical filtering at remote nodes to separate the BB and RF signals. Therefore, the new method will considerably reduce the complexity and improve the performance.

\section{EXPERIMENTAL SETUP AND RESUlTS}

Fig. 1 illustrates the conceptual diagram of the proposed system. A dual-electrode Mach-Zehnder modulator (MZM) is employed to simultaneously generate RF and BB signals. The bias of MZM is set at the quadrature point $V_{\pi} / 2$, where $V_{\pi}$ is the $\pi$ phase shift driving voltage. The electrical OOK RF signal is obtained by mixing a $1.25-\mathrm{Gb} / \mathrm{s}$ data signal with a 5-GHz sinusoidal signal, and the electrical BPSK RF signal is obtained by mixing a $625-\mathrm{Mb} / \mathrm{s}$ data signal with a $14.375-\mathrm{GHz}$ 


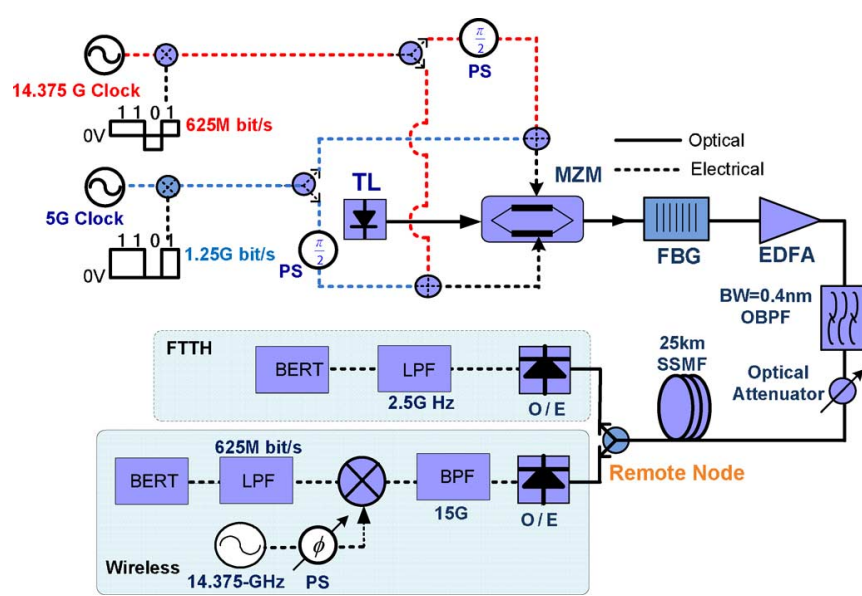

Fig. 2. Experimental setup for RF and BB signal generation and transmission. (PS: phase shifter; FBG: fiber Bragg grating; OBPF: optical bandpass filter; BPF: bandpass filter; LPF: low-pass filter; BERT: BER tester.)

sinusoidal signal. Both the OOK and BPSK RF signals are divided into two identical signals by electrical power dividers. The two driving signals of the dual-electrode MZM are the combination of the divided OOK and BPSK RF signals, except that the BPSK RF signal of the upper arm driving signal is delayed with $90^{\circ}$ phase shift and the OOK RF signal of the lower arm driving signal is delayed with $90^{\circ}$ phase shift.

These two driving signals result in two optical single sideband signals with the generated optical spectrum on the different side of the optical carrier. At the remote nodes, no optical filter is required to separate the BB and RF signals. For FTTH BB applications, the square terms of the RF PSK subcarrier and optical carrier contribute only dc terms to the BB signals after photodiode detection and will cause negligible interference on the OOK BB signal after being removed by an electrical dc block. Thus, the OOK BB signal can be easily recovered with a typical low-speed receiver, whereas the PSK RF signal can be recovered using a high-speed receiver with no interference from the BB signals.

Fig. 2 illustrates the experimental setup: a continuous-wave laser is generated by a tunable laser; the BPSK RF signal is a $625-\mathrm{Mb} / \mathrm{s}$ pseudorandom binary sequence (PRBS) signal with word length of $2^{31}-1$ and up-converted using a $14.375-\mathrm{GHz}$ sinusoidal signal; the OOK RF signal is a $1.25-\mathrm{Gb} / \mathrm{s}$ PRBS with the same word length and up-converted with a $5-\mathrm{GHz}$ sinusoidal signal. After the MZM, a fiber Bragg grating is used to adjust the optical carrier power for receiver sensitivity optimization. The hybrid optical signal is amplified by an erbium-doped fiber amplifier with the optical launched power set at $0 \mathrm{dBm}$ to avoid fiber nonlinearity. After transmission over $25-\mathrm{km}$ standard single-mode fiber (SSMF), the hybrid optical signal is separated for different applications just using an optical coupler. For FTTH BB application, the OOK BB signal is directly detected by using a commercial $1.25-\mathrm{Gb} / \mathrm{s}$ optical receiver. For radio-over-fiber applications, the BPSK $\mathrm{RF}$ signals are detected by using a high-speed photoreceiver, down-converted with $14.375-\mathrm{GHz}$ sinusoidal signal, and then pass through low-pass filters with 3-dB bandwidth of $625 \mathrm{MHz}$.

The optical power ratio between a BPSK subcarrier and an optical carrier is an important parameter which influences the

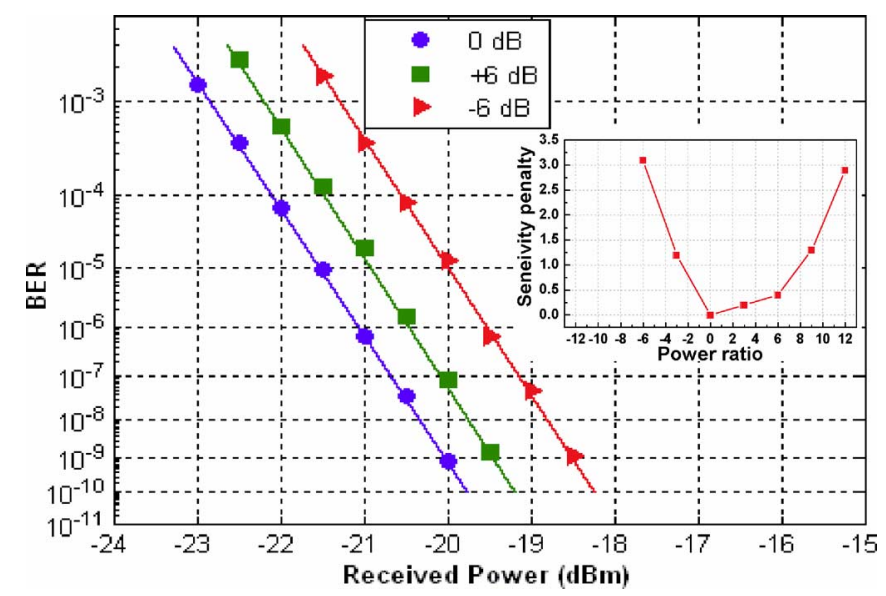

Fig. 3. BER curves of BPSK signals.

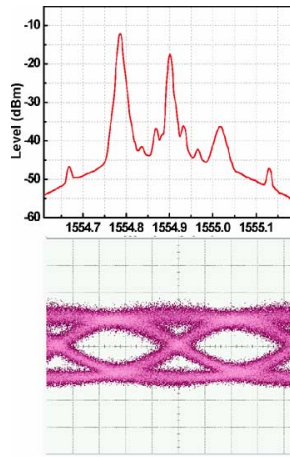

(a)

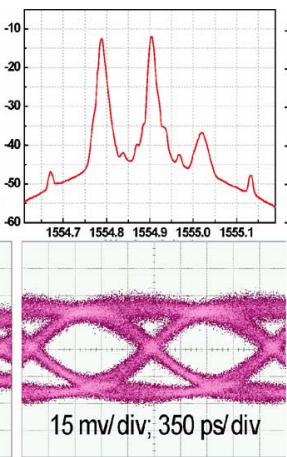

(b)

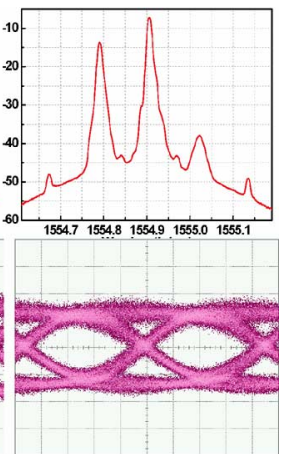

(c)
Fig. 4. Spectra (resolution $0.01 \mathrm{~nm}$ ) and eye diagrams of BPSK signals. (a) $-6 \mathrm{~dB}$; (b) $0 \mathrm{~dB}$; (c) $+6 \mathrm{~dB}$.

receiver sensitivity of BPSK signal. To optimize the RF BPSK receiver sensitivity, the OOK signal is turned OFF first. Only the 14.375-GHz BPSK RF signal is modulated on the optical subcarrier. The system sensitivities are measured with optical carrier to subcarrier power ratio of $-6,0$, and $6 \mathrm{~dB}$. Fig. 3 plots the bit-error-rate (BER) curve for different optical carrier to subcarrier power ratio. The inset within Fig. 3 shows the sensitivity penalties versus the power ratio of optical carrier power to RF BPSK subcarrier power at the BER of $10^{-9}$. The corresponding optical spectra and eye diagrams are shown in Fig. 4.The best receiver sensitivity is obtained when the optical carrier to subcarrier power ratio is $0 \mathrm{~dB}$ and the results agree with a previous published paper [7].

Compared with wireline transmission media (i.e., fiber), wireless signals are more susceptive to the environment (the presence of radio-opaque obstacles, multipath interference, etc.) Therefore, system operators need to be able to freely adjust the power ratio between wireline and wireless services according to geographic variation. The proposed system provides an easily adjustable power splitting scheme to adapt different geographic landscapes. Fig. 5 illustrates the receiver sensitivity of both BPSK RF and BB OOK signals at a BER of $10^{-9}$ with different RF OOK subcarrier to RF BPSK subcarrier optical power ratio $\left(P_{\mathrm{OOK}} / P_{\mathrm{BPSK}}, P_{\mathrm{OOK}}\right.$, and $P_{\mathrm{BPSK}}$ are the powers of RF OOK subcarrier and RF BPSK subcarrier, respectively). A receiver sensitivity tradeoff between the $\mathrm{BB}$ 


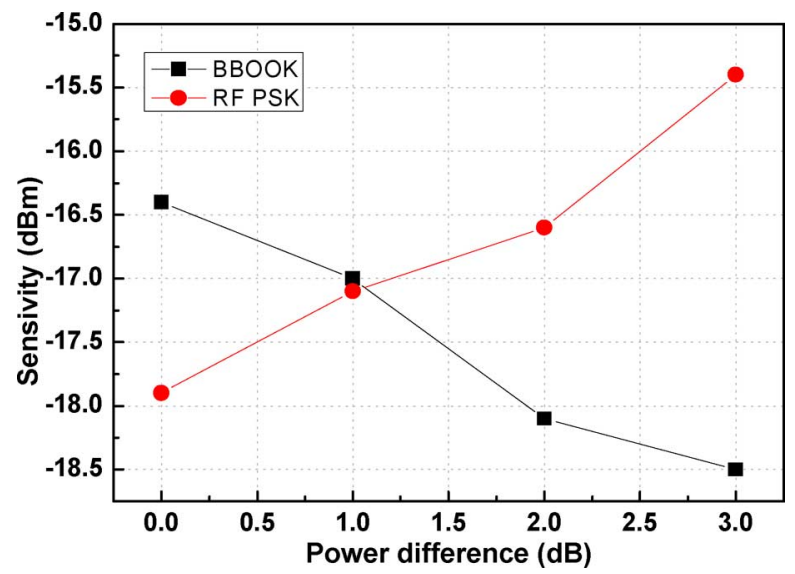

Fig. 5. BB OOK and RF BPSK sensitivities with different RF OOK to RF BPSK optical subcarrier power ratios.

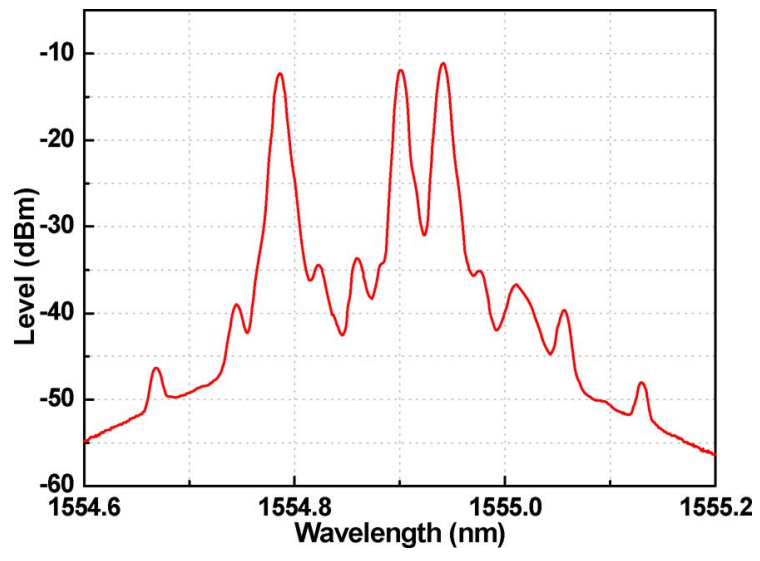

Fig. 6. Optimized BTB optical spectrum. (Resolution: $0.01 \mathrm{~nm}$.)

OOK and down-converted RF BPSK signals is observed. The sensitivities of BB OOK and RF BPSK signals can be adjusted according to the needs of various environments. In this letter, the $\mathrm{BB}$ and $\mathrm{RF}$ signals with the same receiver sensitivities are set as the optimal condition, where the optical power of the OOK subcarrier is $1 \mathrm{~dB}$ higher than that of the RF BPSK subcarrier, as shown in Fig. 6. At the optimal condition, there is about 3-dB penalty when the RF BPSK subcarrier is turned ON or OFF. Among this $3-\mathrm{dB}$ penalty, $1.3 \mathrm{~dB}$ is due to the RF BPSK subcarrier power and $1.7 \mathrm{~dB}$ is due to channel crosstalk and noises.

Fig. 7 shows the receiving sensitivities of both the BB OOK and down-converted RF BPSK signals at $0 \mathrm{~km}$ and following $25-\mathrm{km}$ SSMF transmission. For BB signals, there are three terms of signals after the square-law photodiode detection: the optical carrier, the RF BPSK subcarrier, and the OOK subcarrier. Although the optical carrier and the RF BPSK subcarrier contribute dc power to BB signals, noises of these three carriers accumulate on BB signals after photodiode detection. Therefore, the OOK BB signal is noisier than the RF BPSK signal. However, the sensitivities are still good enough for corresponding applications. The power penalties of both the RF PSK and BB OOK signals at BER of $10^{-9}$ are less than $0.5 \mathrm{~dB}$.

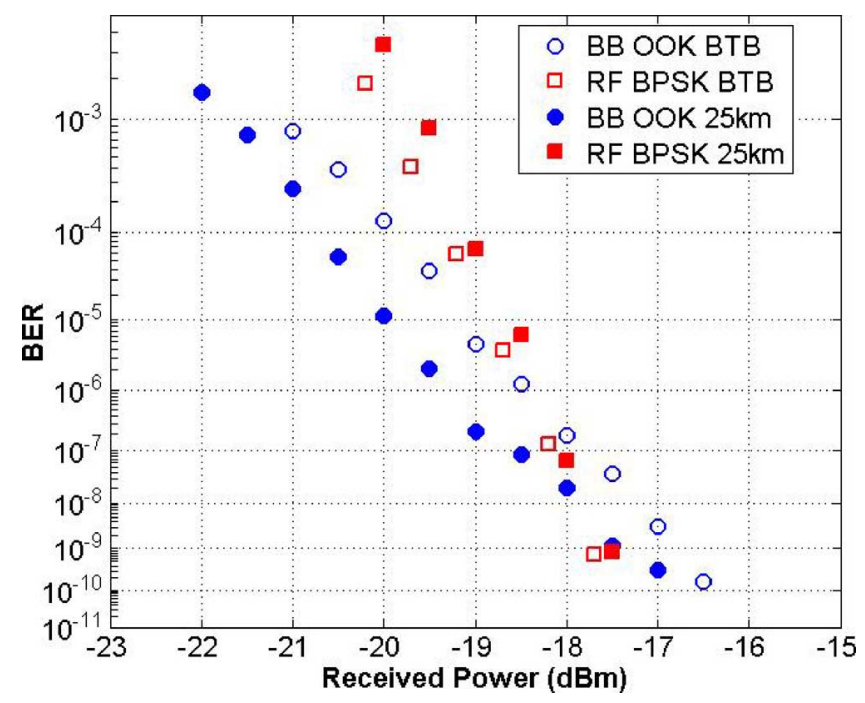

Fig. 7. BER curves of BB OOK and RF BPSK signals.

\section{CONCLUSION}

In this letter, we experimentally demonstrate simultaneous generation and transmission of FTTH BB and wireless RF signals by using a dual-electrode MZM for hybrid access network applications. No optical filter is needed at remote nodes for separating BB and RF signals. The proposed system provides an easily adjustable receiver sensitivity scheme to adapt different geographic landscapes. Following $25 \mathrm{~km}$ of SSMF, the receiver sensitivity penalties of both $\mathrm{RF}$ and $\mathrm{BB}$ signals are less than $0.5 \mathrm{~dB}$. The proposed system provides a simple and cost-effective solution for future broadband multiservice hybrid access networks.

\section{REFERENCES}

[1] T. Kamisaka, T. Kuri, and K. Kitayama, "Simultaneous modulation and fiber-optic transmission of $10-\mathrm{Gb} / \mathrm{s}$ baseband and $60-\mathrm{GHz}-$ band radio signals on a single wavelength," IEEE Trans. Microw. Theory Tech., vol. 49, no. 10, pp. 2013-2017, Oct. 2001.

[2] K. Ikeda, T. Kuri, and K. Kitayama, "Simultaneous three-band modulation and fiber-optic transmission of $2.5-\mathrm{Gb} / \mathrm{s}$ baseband, microwave-, and 60-GHz-band signals on a single wavelength," J. Lightw. Technol., vol. 21, no. 12, pp. 3194-3202, Dec. 2003.

[3] G. K. Chang, J. Yu, Z. Jia, and J. Yu, "Novel optical-wireless access network architecture for simultaneously providing broadband wireless and wired services," in Proc. Opt. Fiber Commun. (OFC), Anaheim, CA, 2006, Paper OFM1.

[4] J. Yu, Z. Xu, X. Zhang, Z. Jia, T. Wang, and G. K. Chang, "A cost-effective scheme to generate and de-multiplex multiple frequency millimeter-wave signals in a ROF network," in Proc. Eur. Conf. Opt. Commun. (ECOC), Berlin, Germany, 2007, Paper 3.3.3.

[5] Z. Jia, J. Yu, A. Chowdhury, G. Ellinas, and G. K. Chang, "Simultaneous generation and delivery of independent wired and wireless services in radio-over-fiber systems using a single modulator," in Proc. Eur. Conf. Opt. Commun. (ECOC), Berlin, Germany, 2007, Paper 3.3.2.

[6] Q. Chang and Y. Su, "A radio over fiber system for simultaneous generation and transmission of multiband signals," in Proc. Eur. Conf. Opt. Commun. (ECOC), Berlin, Germany, 2007, Paper P091.

[7] C. Lim, C. Lin, M. Attygalle, A. Nirmalathas, D. Novak, and R. Waterhouse, "Analysis of optical carrier-to-sideband ratio for improving transmission performance in fiber-radio links," IEEE Trans. Microw. Theory Tech., vol. 54, no. 5, pp. 2181-2187, May 2006. 